\title{
Nursing workload in relation to nosocomial infection in public hospital Intensive Care Unit, Malaysia
}

\begin{abstract}
A Nosocomial infection (NIs) is an infection acquired in the hospital and becomes evident 48 hours after admission to the hospital. It continues to be a major problem affecting patient safety and quality of care in Intensive Care Unit (ICU).One of the most pertinent factors leading to NIs is nursing workload. The aim of the study is to determine nursing workload on NIs rate in the ICU, Hospital Serdang. A retrospective cross sectional study was used. Data were collected from NIs surveillance, staff nurses' duty roster and patient census by using a performa. The result in this study shows, $19 \%(n=88)$ cases of NIs were detected in $12 \%($ $\mathrm{n}=57$ ) of patient. There was significant moderate positive relationship between the number of infection and the total number of nurses working overtime $(\mathrm{r}=0.66, \mathrm{n}=88, \mathrm{p}=0.001)$ and the number of infection and the total number of new staffs $(r=0.51, n=88, p=0.001)$. There was significant weak positive relationship between the number of infection and the total number of nurses taking medical leave or emergency leave $(r=0.33, n=88, p=0.001)$. This study provide a baseline to improve nursing staffing in ICU. Future planning of ICU staffing should be comprehensive so that patients are not disadvantaged.
\end{abstract}

Keyword: Phenolic compounds; Antioxidant activity; FRAP; Total phenolic content; Reducing power 